\title{
Accelerated green process of 2,5-dimethylpyrazine production from glucose by genetically modified Escherichia coli
}

Jianzhong Xu ${ }^{\text {a,1,*, Haibo Yu }}{ }^{\text {a,1, }}$, Xiulai Chen ${ }^{\text {b, Liming Liu }}{ }^{\text {b,*, Weiguo Zhang }}{ }^{\text {a }}$

a The Key Laboratory of Carbohydrate Chemistry and Biotechnology, Ministry of Education, School of Biotechnology, Jiangnan University, 1800\# Lihu Road, WuXi 214122, People’s Republic of China

b State Key Laboratory of food Science and Technology, School of Biotechnology, Jiangnan University, 1800\# Lihu Road, WuXi 214122, People’s Republic of China

${ }^{1}$ These authors contributed equally to this work.

* Corresponding author: Jian-Zhong Xu, Tel: +86-510-85329312; Fax: +86-510-85329312; E-mail: xujianzhong@jiangnan.edu.cn; Li-Ming Liu, Tel: +86-510-85329312; Fax: +86-510-85329312; E-mail: $\underline{\text { liulm jn@sina.com }}$ 


\section{DNA manipulations and transformations}

The plasmids and oligonucleotides used in this study are listed in Table 1 and Table S1, respectively. Chromosomal DNA was extracted from E. coli using an Ezup Bacterial Genomic DNA Extraction Kit according the protocol supplied by the manufacturer (Sangon, Shanghai, China). The target gene segments were synthesized after codon optimization by GENEWIZ, Inc. (Suzhou, China). The build processes of plasmids and strains are illustrated as follows. Plasmids were extracted from E. coli using the SanPrep Mini Plasmid Kit (Sangon, Shanghai, China) and confirmed by restriction endonuclease reaction. The plasmid transformation was performed according to the descriptions of previous reports ${ }^{l}$.

\section{The build processes of recombinant plasmids}

The plasmid pEC-XK99E- $t d h_{\mathrm{X}}$ construction

The nucleotide sequences of the TDH-coding gene $t d h$ from different strains with the RBS of $E$. coli and two endonucleases (i.e., EcoRI and BamHI) were synthetized after codon optimization by GENEWIZ, Inc. (Suzhou, China), and then linked into an expression plasmid pEC-XK99E. The resulted plasmid was designated as pEC-XK99E- $t d h_{\mathrm{X}}\left({ }_{\mathrm{x}}\right.$ represents the different strains).

The plasmid pEC-XK99E- $t d h_{\mathrm{Ec}}-n o x E_{\mathrm{Lc}}$ construction

The nucleotide sequences of the NoxE-coding gene noxE from Lactococcus cremoris MG1363 with two endonucleases (i.e., XbaI and HindIII) were synthetized after codon optimization by GENEWIZ, Inc. (Suzhou, China), and then linked into a recombinant plasmid pEC-XK99E-tdh $h_{\mathrm{Ec}}$. The resulted plasmid was designated as pEC-XK99E- $t d h_{\mathrm{Ec}}-n o x E_{\mathrm{Lc}}$. 
The plasmid pEC-XK99E-tdh $h_{\mathrm{Ec}}-\mathrm{P} n o x E_{\mathrm{Lc}}$ construction

The nucleotide sequences of the NoxE-coding gene noxE from Lactococcus cremoris MG1363 with the RBS of E. coli and promoter $\mathrm{P}_{\text {trc }}$ from plasmid pEC-XK99E were synthetized after codon optimization by GENEWIZ, Inc. (Suzhou, China), and then linked into a recombinant plasmid pEC-XK99E- $t d h_{\mathrm{Ec}}$ under the terminator T2. The resulted plasmid was designated as pEC-XK99E- $t d h_{\mathrm{Ec}}-\mathrm{P} n o x E_{\mathrm{Lc}}$.

The plasmid pEC-XK99E- $t d h_{\mathrm{Ec}}-a a o_{\mathrm{So}}-\mathrm{P} n o x E_{\mathrm{Lc}}$ construction

The nucleotide sequences of the AAO-coding gene aao from Streptococcus oligofermentans with two endonucleases (i.e., $X b a \mathrm{I}$ and $P s t \mathrm{I}$ ) were synthetized after codon optimization by GENEWIZ, Inc. (Suzhou, China), and then linked into a recombinant plasmid pEC-XK99E- $t d h_{\mathrm{Ec}}-\mathrm{P} n o x E_{\mathrm{Lc}}$. The resulted plasmid was designated as pEC-XK99E-tdh $h_{\mathrm{Ec}}-a a o_{\mathrm{So}}-\mathrm{P} n o x E_{\mathrm{Lc}}$.

The plasmid pEC-XK99E-tdh $h_{\mathrm{Ec}}-\mathrm{P} a a o_{\mathrm{So}}-\mathrm{P} n o x E_{\mathrm{Lc}}$ construction

The nucleotide sequences of the AAO-coding gene aao from Streptococcus oligofermentans with the RBS of E. coli, promoter $\mathrm{P}_{\text {trc }}$ from plasmid pEC-XK99E and two endonucleases (i.e., $X b a \mathrm{I}$ and $P s t \mathrm{I}$ ) were synthetized after codon optimization by GENEWIZ, Inc. (Suzhou, China), and then linked into a recombinant plasmid pEC-XK99E- $t d h_{\mathrm{Ec}}-\mathrm{P} n o x E_{\mathrm{Lc}}$. The resulted plasmid was designated as pEC-XK99E- $t d h_{\mathrm{Ec}}-\mathrm{P} a a o_{\mathrm{So}}-\mathrm{P} n o x E_{\mathrm{Lc}}$. The plasmid pCDFDuet- $\mathrm{P}_{\text {sod }}-s s t T_{\mathrm{Ec}}$ construction

The nucleotide sequences of the SstT-coding gene sst $T$ from E. coli MG1655 with the RBS of E. coli, promoter $\mathrm{P}_{\text {sod }}$ and two endonucleases (i.e., EcoRI and HindIII) were synthetized after codon optimization by GENEWIZ, Inc. (Suzhou, China), and then linked into an expression plasmid pCDFDuet-1. The resulted plasmid was designated as pCDFDuet- $\mathrm{P}_{\text {sod }}-S s t T_{\mathrm{Ec}}$. 
The plasmid pCDFDuet- $\mathrm{P}_{\text {sod }}-S s t T_{\mathrm{Ec}}$ construction

The nucleotide sequences of the SstT-coding gene sstT from E. coli MG1655 with the RBS of E. coli, promoter $\mathrm{P}_{\mathrm{vgb}}$ and two endonucleases (i.e., EcoRI and HindIII) were synthetized after codon optimization by GENEWIZ, Inc. (Suzhou, China), and then linked into an expression plasmid pCDFDuet-1. The resulted plasmid was designated as pCDFDuet- $\mathrm{P}_{\mathrm{vgb}}-s s t T_{\mathrm{Ec}}$.

The procedures of recombinant strain constructions

Construction of T6-47-1, T6-47-2, T6-47-3, T6-47-4 and T6-47-5

The plasmid pEC-XK99E- $t d h_{\mathrm{Ec}}, \quad$ pEC-XK99E- $t d h_{\mathrm{Ec}}-n o x E_{\mathrm{Lc}}, \quad \mathrm{pEC}-\mathrm{XK} 99 \mathrm{E}-t d h_{\mathrm{Ec}}-\mathrm{P} n o x E_{\mathrm{Lc}}$, pEC-XK99E- $t d h_{\mathrm{Ec}}-a a o_{\mathrm{So}}-\mathrm{P} n o x E_{\mathrm{Lc}}$ and pEC-XK99E- $t d h_{\mathrm{Ec}}-\mathrm{Paao} \mathrm{So}_{\mathrm{S}}-\mathrm{P} n o x E_{\mathrm{Lc}}$ were transformed into T6-47, respectively, and then the resulting recombinant strain was designated as T6-47-1, T6-47-2, T6-47-3, T6-47-4 and T6-47-5, respectively.

Construction of T6-47-6 and T6-47-7

The plasmid pCDFDuet- $\mathrm{P}_{\mathrm{sod}}-S s t T_{\mathrm{Ec}}$ and pCDFDuet- $\mathrm{P}_{\mathrm{vgb}}-s s t T_{\mathrm{Ec}}$ were transformed into T6-47-4, respectively, and then resulting in T6-47-6 and T6-47-7, respectively. 
Table S1 The oligonucleotides used in this study

\begin{tabular}{|c|c|c|c|}
\hline Primer & Sequence (5'-3') & Cleavage sites & Purposes \\
\hline$t d h-\mathrm{F}$ & CGGGATCCGATGAAAGCGTTATCC & BamHI & \multirow{4}{*}{$\begin{array}{l}\text { Obtaining the gene of } t d h \text { from the } \\
\text { genome of } E . \text { coli } \mathrm{K}-12 \text { and } \\
\text { verifying the existence of } t d h \text { in } E \text {. } \\
\text { coli } \text { T6- } 47 \text {. }\end{array}$} \\
\hline & AAACT & & \\
\hline$t d h-\mathrm{R}$ & GGAATTCTTAATCCCAGCTCAGAAT & EcoRI & \\
\hline & AACT & & \\
\hline$t d h_{\mathrm{in}}-\mathrm{F}$ & ATAACTGGGATGAGTGGT & - & \multirow{2}{*}{$\begin{array}{l}\text { Verifying the existence of } t d h \text { in } E \text {. } \\
\text { coli T6- } 47 \text {. }\end{array}$} \\
\hline$t d h_{\mathrm{in}}-\mathrm{R}$ & CATGGTGTCAAGCATGGT & - & \\
\hline $\operatorname{sstT}_{\mathrm{RT}}-\mathrm{F}$ & GTGATGGCATCTATTGCT & - & \multirow{2}{*}{ RT-PCR for $s s t T$} \\
\hline $\operatorname{sstT}_{\mathrm{RT}}-\mathrm{R}$ & ATATCACCCGCGCTACT & - & \\
\hline $16 \mathrm{~S}-\mathrm{F}^{\mathrm{r}}$ & GCTCGTGTTGTGAAATGTTG & - & \multirow{2}{*}{ RT-PCR for $16 \mathrm{~S}$ rRNA } \\
\hline $16 \mathrm{~S}-\mathrm{R}^{\mathrm{r}}$ & GTAAGGGCCATGATGACTTG & - & \\
\hline
\end{tabular}

${ }^{a}$ The letters with underline represent cleavage sites and the bold letters represent the homologous sequence of the target genes; -: No cleavage sites. 
a

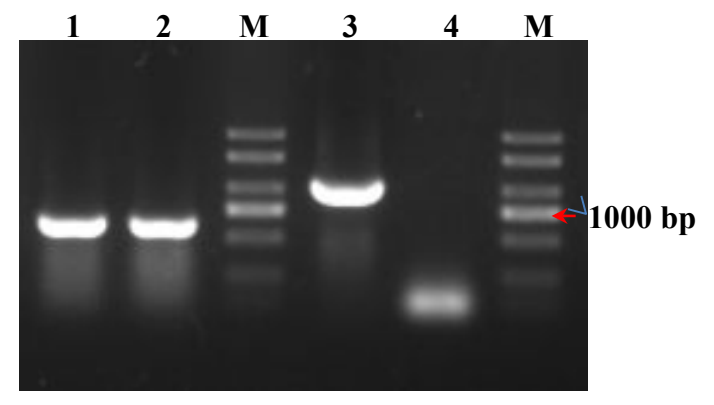

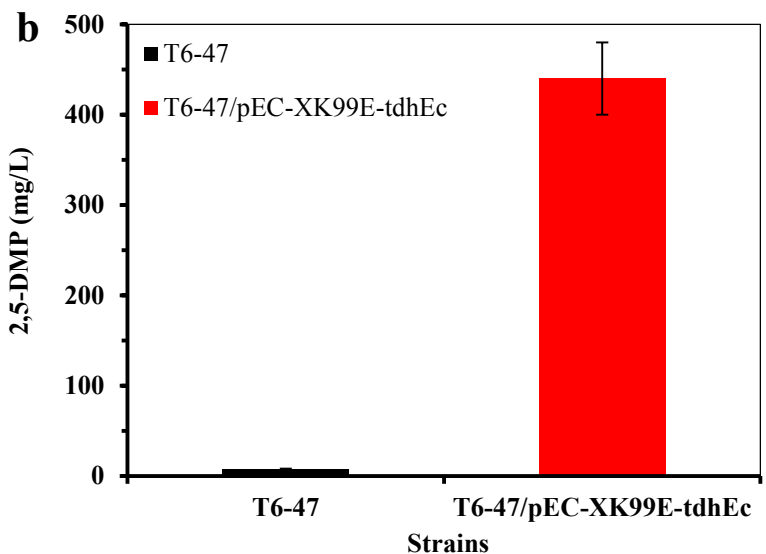

Fig. S1. Analyzing the TDH-coding gene $t d h$ in strains MG1655 and T6-47 by PCR (a) and by re-overexpression of TDH from strain MG1655 (b). In Fig. S1a, Lane 1 strain MG1655 using $t d h_{\mathrm{in}}-\mathrm{F}$ and $t d h_{\mathrm{in}}-\mathrm{R}$ as primer pairs; Lane 2 strain T6-47 using $t d h_{\mathrm{in}}-\mathrm{F}$ and $t d h_{\mathrm{in}}-\mathrm{R}$ as primer pairs; Lane 3 strain MG1655 using $t d h-\mathrm{F}$ and $t d h-\mathrm{R}$ as primer pairs; Lane 4 strain T6-47 using $t d h-\mathrm{F}$ and $t d h-\mathrm{R}$ as primer pairs; Lane $M$ DNA marker. The standard errors are shown as bars. 


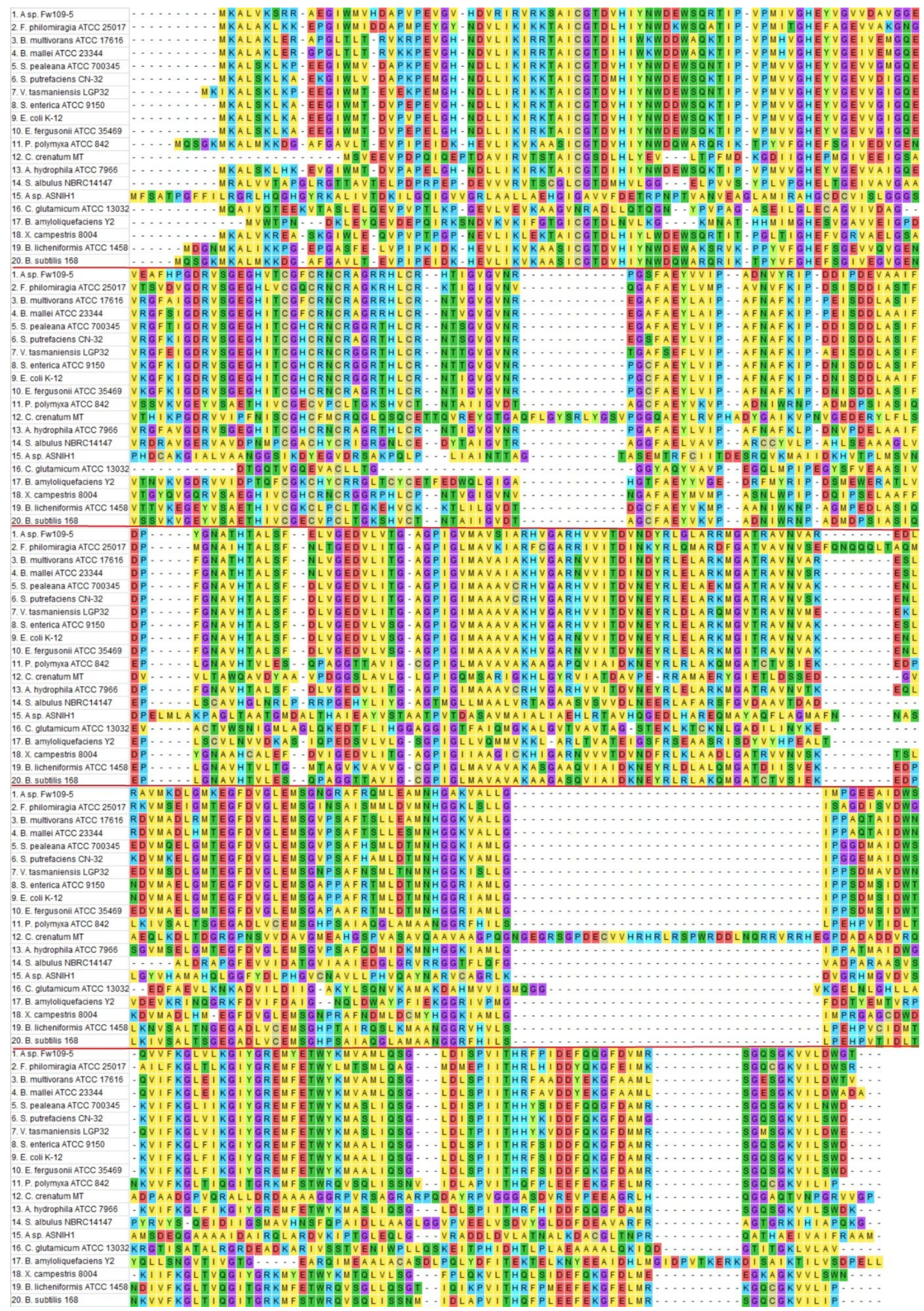

Fig. S2. Alignment of amino acid sequences of TDH proteins from 20 representative strains. 


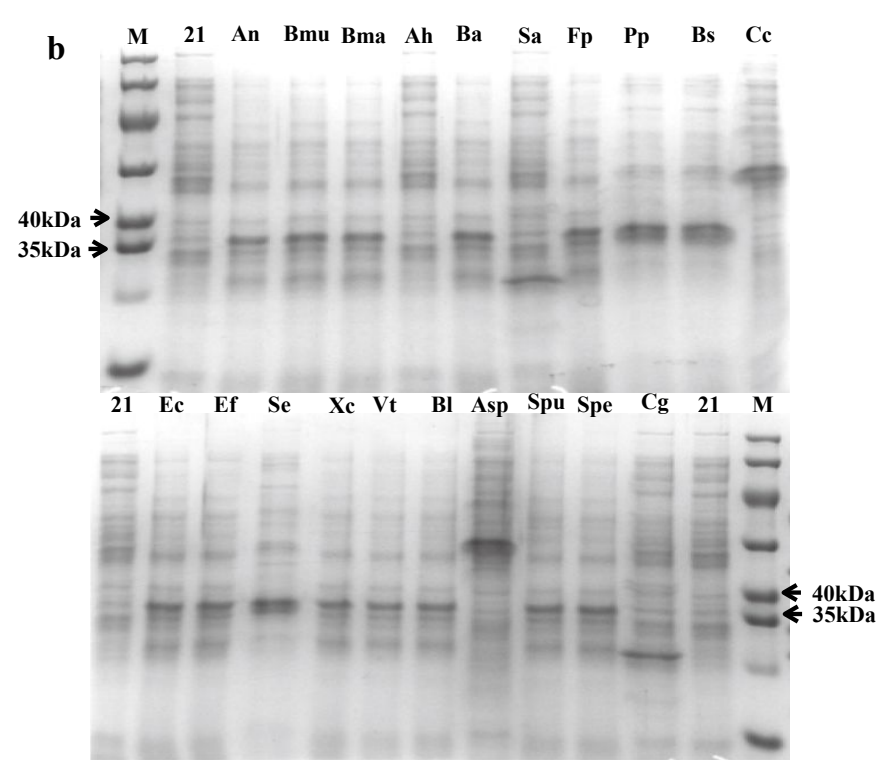

Fig. S3. Analyzing the expression of TDH from different strain by SDS-PAGE. Ah Aeromonas hydrophica ATCC7966, Asp A. sp. ASNIH1, An Anaeromyxobacter sp. Fw109-5, Bmu Burkholderia multivorans ATCC17616, Bma B. mallei ATCC23344, B1 Bacillus licheniformis ATCC14580, Ba B. amyloliquefaciens Y2, Bs B. subtilis 168, Cg Corynebacterium glutamicum ATCC13032, Ce C. crenatum MT, Fp Francisella philomiragia ATCC25017, Pp Paenibacillus polymyxa ATCC842,Sa Streptomyces albulus NBRC14147, Spe Shewanella pealeana ATCC700345, Spu S. putefaciens CN-32, Se Salmonella enterica ATCC9150, Vt Vibrio tasmaniensis LGP32, Xc Xanthomonas campestris 8004, Ef Escherichia fergusonli ATCC35469, Ec E. coli MG1655 


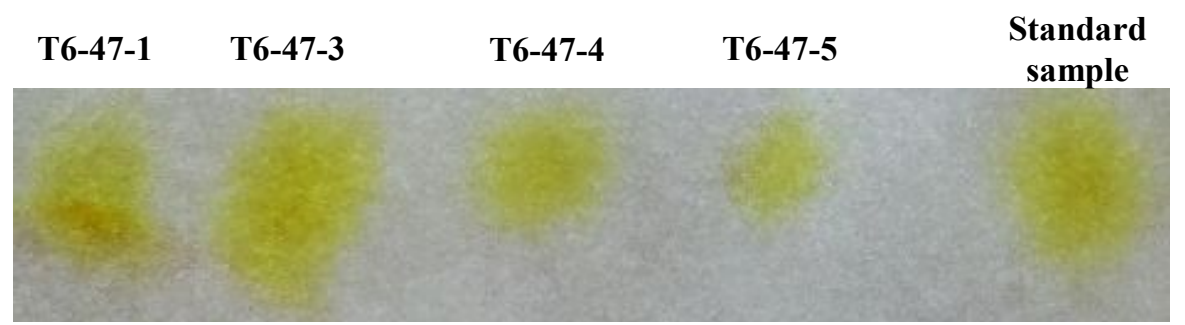

Fig. S4. Comparison of aminoacetone accumulation in strains T6-47-1, T6-47-3, T6-47-4 and T6-47-5 by paper chromatography. 

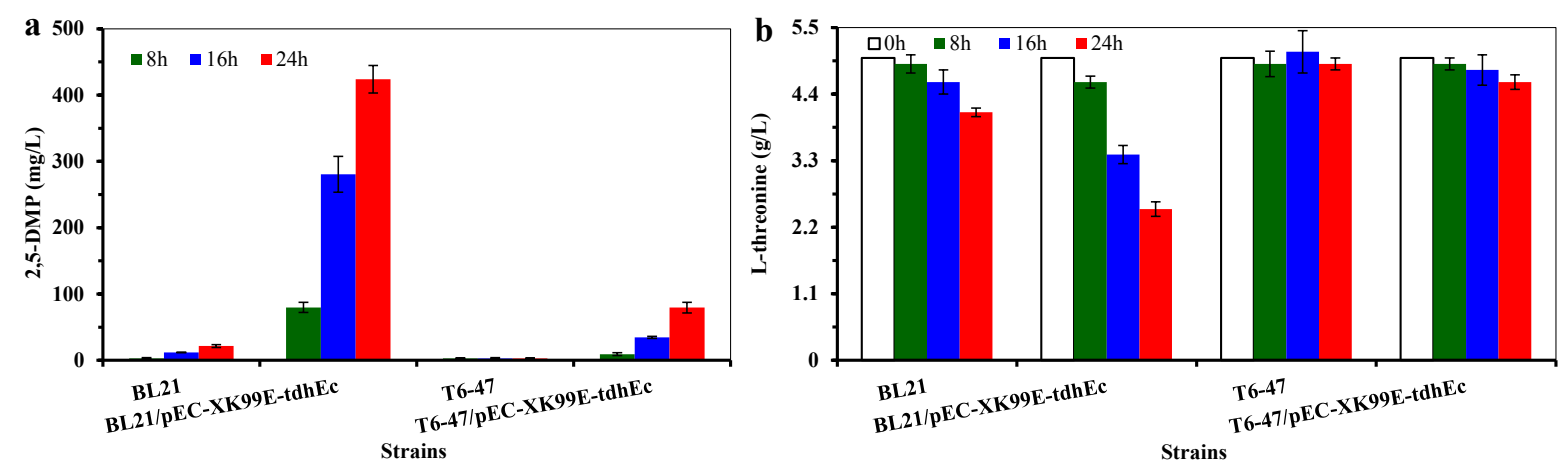

Fig. S5. The 2,5-DMP production (a) and L-threonine consumption (b) of E. coil strains BL21, BL21/pEC-XK99E- $t d h_{\mathrm{Ec}}, \mathrm{T} 6-47$ and T6-47/pEC-XK99E- $t d h_{\mathrm{Ec}}$ by whole-cell biocatalyst. The standard errors are shown as bars. 

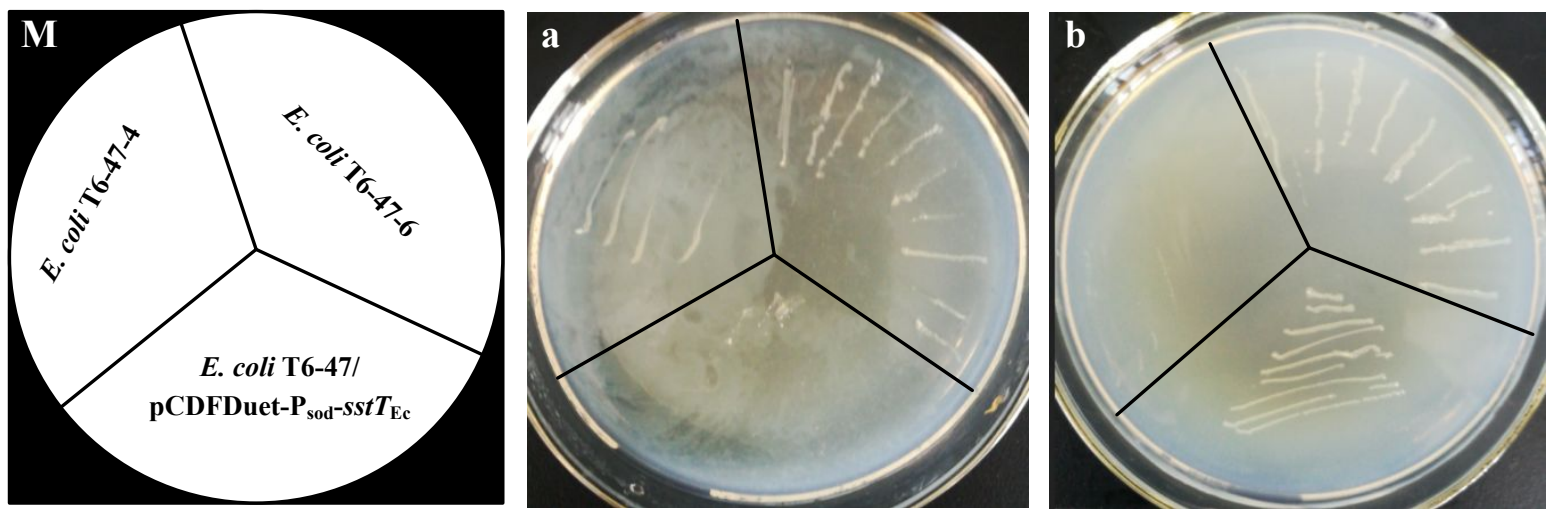

Fig. S6. Analyzing the plasmid co-existence between plasmids pEC-XK99E-tdh $h_{\mathrm{Ec}}-\mathrm{P} n o x E_{\mathrm{Lc}}-a a o_{\mathrm{So}}$ and pCDFDuet- $\mathrm{P}_{\text {sod }}-S s t T_{\mathrm{Ec}}$ in strain T6-47-3. Fig. M represents the location of strains in Fig. a and Fig. b; Fig. a represents the LB medium with $50 \mu \mathrm{g} / \mathrm{mL}$ of kanamycin; Fig. b represents the LB medium with $50 \mu \mathrm{g} / \mathrm{mL}$ of streptomycin. 


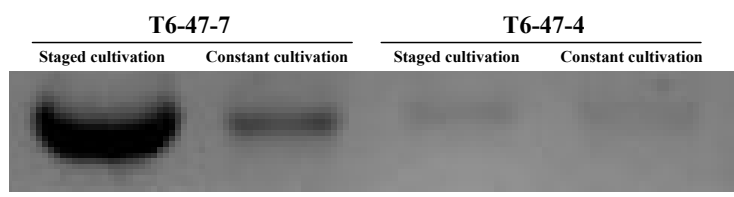

Fig. S7. Analyzing the expression levels of gene $s s t T$ from strains T6-47-4 and T6-47-7 by semiquantitative RT-PCR during staged cultivation and constant cultivation. 


\section{Supplementary References}

[1] Xu, J. Z., Han, M., Zhang, J. L., Guo, Y. F., Qian, H., and Zhang, W. G. (2014) Improvement of L-lysine production combines with minimization of by-products synthesis in Corynebacterium glutamicum, $J$ Chem Technol Biot 89, 1924-1933. 